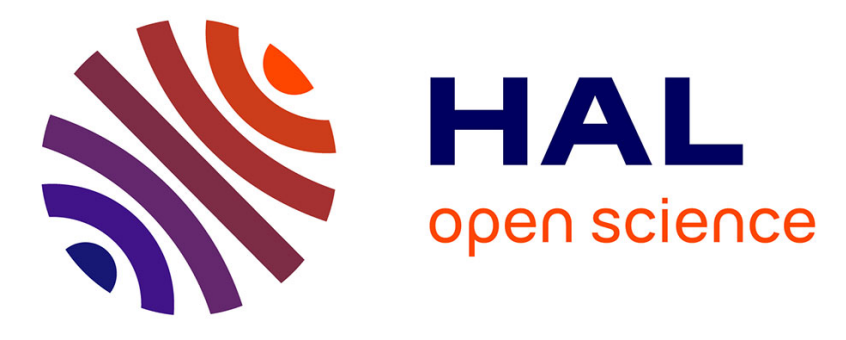

\title{
Imaging Photoplethysmography: Signal Waveform Analysis
}

\author{
Djamaleddine Djeldjli, Frédéric Bousefsaf, Choubeila Maaoui, Fethi \\ Bereksi-Reguig
}

\section{- To cite this version:}

Djamaleddine Djeldjli, Frédéric Bousefsaf, Choubeila Maaoui, Fethi Bereksi-Reguig. Imaging Photoplethysmography: Signal Waveform Analysis. 2019 10th IEEE International Conference on Intelligent Data Acquisition and Advanced Computing Systems: Technology and Applications (IDAACS), Sep 2019, Metz, France. pp.830-834, 10.1109/IDAACS.2019.8924239 . hal-03352098

\section{HAL Id: hal-03352098 \\ https://hal.science/hal-03352098}

Submitted on 22 Sep 2021

HAL is a multi-disciplinary open access archive for the deposit and dissemination of scientific research documents, whether they are published or not. The documents may come from teaching and research institutions in France or abroad, or from public or private research centers.
L'archive ouverte pluridisciplinaire HAL, est destinée au dépôt et à la diffusion de documents scientifiques de niveau recherche, publiés ou non, émanant des établissements d'enseignement et de recherche français ou étrangers, des laboratoires publics ou privés. 


\title{
Imaging Photoplethysmography: Signal Waveform Analysis
}

\author{
Djamaleddine Djeldjli ${ }^{1}$, Frédéric Bousefsaf ${ }^{2}$, Choubeila Maaoui ${ }^{3}$, Fethi Bereksi-Reguig ${ }^{4}$ \\ 1,2,3 LCOMS - Université de Lorraine, 7 rue Marconi, 57070 Metz, France \\ \{djamaleddine.djeldjli, frederic.bousefsaf, choubeila.maaoui\}@univ-lorraine.fr \\ ${ }^{1,4}$ Laboratoire de Génie Biomédical -Université de Tlemcen, 13000 Tlemcen, Algérie, bereksif@yahoo.fr
}

\begin{abstract}
Imaging photoplethysmography (iPPG) is used to remotely measure the blood volume pulse using a camera. Traditionally, it has been employed to estimate heart and respiratory rates, and recently blood pressure and oxygen saturation. Biomedical engineering researchers have become increasingly interested in IPPG because of its several advantages as comfort, non-invasive, simple, nonstressful and inexpensive characteristics. Moreover, the results of the waveform signal analysis obtained by the conducted studies on contact PPG sensors made the use of iPPG a more promising technique for early screening of various atherosclerotic and cardiovascular pathologies. iPPG waveform has not been studied before. In this paper we process the iPPG signal obtained using a camera to extract its waveform features such as areas and time parameters. These features will make possible a non-contact estimation of arterial stiffness and early diagnosis of many cardiovascular pathologies. The obtained results show a high correlation with those obtained by an approved contact PPG sensor.
\end{abstract}

Keywords-photoplethysmography; pulse waveform; signal processing; camera; facial video processing.

\section{INTRODUCTION}

Photoplethysmography (PPG) is an optical noninvasive technique used to determine blood volume changes in the microvascular bed of tissue [1]. The PPG technology has been used in wide range of commercialized medical devices for the assessment of heart rate, oxygen saturation, blood pressure and cardiac output, autonomic function and also detection of peripheral vascular pathologies [2]-[4]. However, in some critical cases, such as burns, traumas, contagious diseases and severe infections, the use of contact PPG sensor is not always possible. Also contact sensors interfere with the subject by producing a psychological stress which can affect the measurement. These various limitations of contact PPG make the use of contactless photoplethysmography more attractive for the measurement and the monitoring of cardiovascular data.

Imaging photoplethysmography allows to determine physiological data such as blood volume pulse remotely, without any skin contact. This is achieved by the analysis of facial video based on subtle momentary changes in the subject's skin color which are not noticeable to the human eye. iPPG has recently attracted increasing interest especially in cardiac activity [5], [6] and respiratory monitoring [7], [8] driven by the demand for low cost, simple, portable, comfortable and non-stressful technology. It was also used for the assessment of mental stress [9], oxygen saturation [10]-[12] and blood pressure measurements [13]-[15].

In this paper we present a particular pulse waveform analysis based on the detection of iPPG from videos recorded by a camera. The paper is organized as follow: a first background section which describes the basics of PPG and iPPG, their applications, limits and clinical significances. In the second section we present the proposed approach which is focused on the measurement of iPPG signal using a camera, its processing and the relevant waveform features extraction. Finally, results are presented and discussed before the last section, where we present conclusion and perspectives about this work.

\section{BACKGROUND}

Currently, to our knowledge, no systematic study has been carried out in existing literature on iPPG signal waveform analysis. For this reason, we review the existing works on contact PPG waveform features and their applications.

PPG waveform is affected by heartbeat, hemodynamic properties and physiological conditions caused by the change in the properties of arterioles. Such effect can be noticed as distortion in the wave profiles [16]. Its analysis is very interesting especially in blood circulation assessment [17] and respiratory monitoring [18].

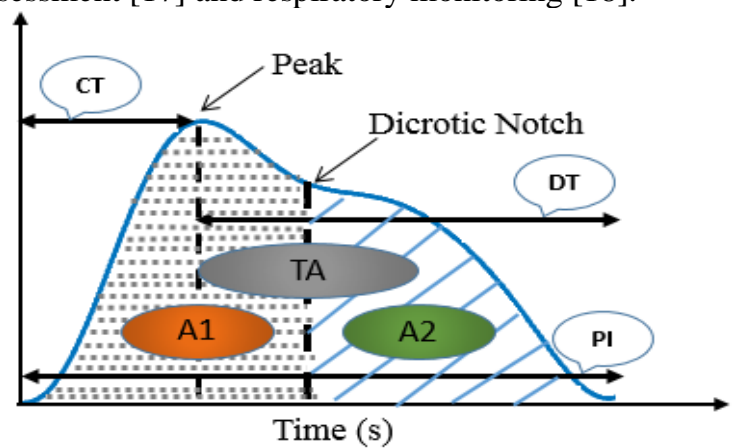

Figure 1. Original PPG waveform features, CT: Crest time, PI: Pulse interval, DT: Diastolic time, TA: Total area, A1: dotted area and A2: Area with blue lines. 
Fig. 1. represents a PPG signal wave of a healthy subject. It was analyzed to extract several features by its second derivative. Initially, Ozawa [19] introduced the use of first and second derivatives to facilitate the interpretation of the original PPG waves. The PPG parameters shown in Fig. 1 can be listed as follows:

1. Crest time (CT) is the time from the foot of the PPG wave to the systolic peak [20]-[22]. Alty et al proved the CT can be an useful variable for cardiovascular disease classification[20]. It details the early systolic phase, which is due to the fast injection of blood during systole. CT increases when vascular blood flow resistance increases [14] .

2. Pulse interval (PI) is the time from the starting to the ending points of PPG waveform. It can be used for heart pulse rate measurement instead of the peak to peak interval [16]. The ratio between pulse interval and pulse amplitude provide an understanding of cardiovascular system properties [25]. It is strongly correlated to the assessment of heart rate variability compared to R-R interval of ECG [26].

3. Diastolic time (DT) corresponds to the time from the PPG waveform peak to its end.

4. Pulse area (PA) is calculated as the total area under the waveform curve [16], [21], [24]. The blood vessel elasticity, peripheral resistance and physiological changes in ejection function modify the total pulse area [21]. It also decreases with exercise [27]. Seitsonen et al. [28] found the PPG total area response to skin incision to differ between movers and non-movers.

5. Area 1 (A1) is the area measured under the curve from the starting point to the dicrotic notch [21], [24]. It mainly reflects the systolic pulse wave characteristics, which are influenced by cardiac ejection function [29].

6. Area 2 (A2) is the area measured under the curve from the dicrotic notch to the end of the waveform [21], [24]. It reflects the diastolic pulse characteristics which are affected by peripheral resistance and arterial compliance [30].

\section{MATERIALS AND METHODS}

\section{A. Materials}

In this study, a fast color Camera (Edmund Optics EO-2223C) is used to record images at nominally 125 frames per second (fps) with $640 \times 480$ pixels resolution. The three RGB channels are encoded with 8 bits per pixel. Also, the illumination is controlled through two LEDs panels (Neewer NL480). Light intensity is fixed at $80 \%$ from a maximum of $3360 \mathrm{lux} / \mathrm{m}$ and color temperature at $3750 \mathrm{~K}$. It is important to note that exposure and white balance have been fixed in these experiments. White balance locally regulates colors and generates non-desired artifacts in webcam PPG signals [7]. For results validation, an approved contact PPG sensor (BVP-Flex/Pro by Thought Technologies Ltd.) was used to record the earlobe PPG signal at a sampling rate of $256 \mathrm{~Hz}$.

\section{B. iPPG signal extraction}

The overall system is composed with both image and signal processing. The fast camera parameters such as zoom, brightness, contrast and color saturation are controlled by the developed algorithm to save a sequence of subject facial images for 60 seconds. Then image processing operations are applied to isolate the forehead pixels used as region of interest (ROI) for the extraction of iPPG signals. The forehead and cheeks areas are rarely covered by clothing or facial hair and correspond to ROI with significant signal to noise ratio [31], [32]. Only the green channel of the RGB forehead images is used for signal extraction. Oxy- and deoxyhemoglobin have a better wavelengths absorption coefficient between 540 and $577 \mathrm{~nm}$, which correspond to yellow/green colors wavelengths [33]. The raw iPPG signal is extracted using a spatial average which transforms a set of pixels into a scalar on green channel forehead image [7]. This operation is applied on a set of $n$ frames to generate $n$ scalar points succession which correspond to the raw iPPG signal, as given in Fig. 2.

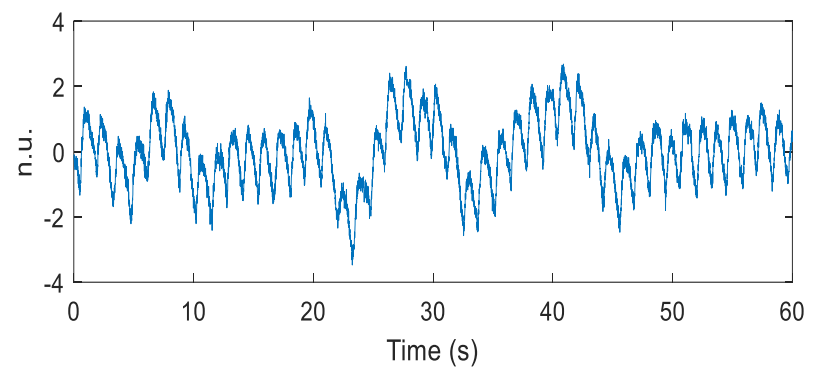

Figure 2. Forehead raw iPPG signal obtained using camera

Raw traces are firstly detrended using a technique based on a smoothness priors approach [34] then interpolated to $256 \mathrm{~Hz}$ to get regular sampling rate. The resulting iPPG signal was denoised by the use of a Butterworth pass band filter. The cutoff frequencies range is comprised between 0.5 and $3 \mathrm{~Hz}$ in order to facilitate the feet and peaks detection (see Fig. 3). We note that the PPG module delivers a filtered PPG signal.

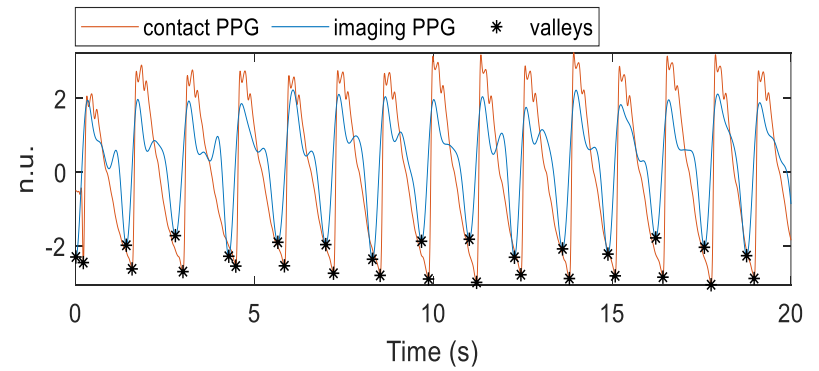

Figure 3. Raw PPG signal (orange) and filtered PPGi (blue), the black markers correspond to the detected valleys 


\section{Waveform features extraction}

Both the filtered iPPG and the contact PPG (ground truth) signals undergo the same processing. We compute, for each pulse wave, several features (see section II). The waves were cut according to the valleys (signal feet) detection as shown in Fig. 3. A wave corresponds to the curve located between two successive signal feet. Their amplitudes were normalized between 0 and 1 because of the amplitude of contact PPG is greater than iPPG and this leads to a large differences in areas calculation. In addition, and to improve features stability, we average every 10 successive pulse waves (moving window of 10 waves for a step of one wave). The dicrotic notch has been localized by a detection of the central maximum in the second derivative of each pulse wave [19], as shown in Fig.4 for iPPG and in Fig.5 for PPG waveforms. The extraction method of each features has been detailed in section II.

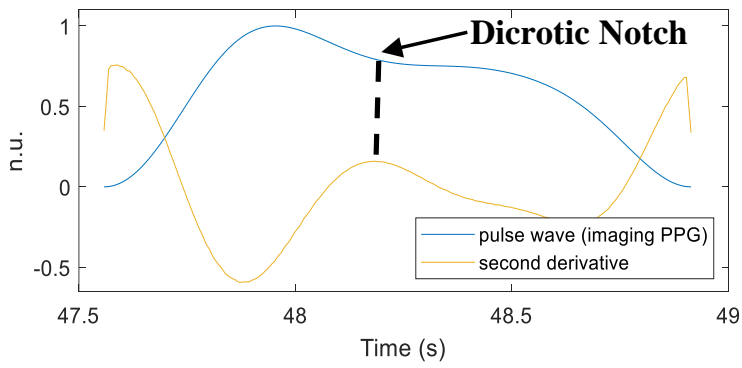

Figure 4. iPPG dicrotic notch detection, blue trace is the iPPG waveform and yellow trace its second derivative.

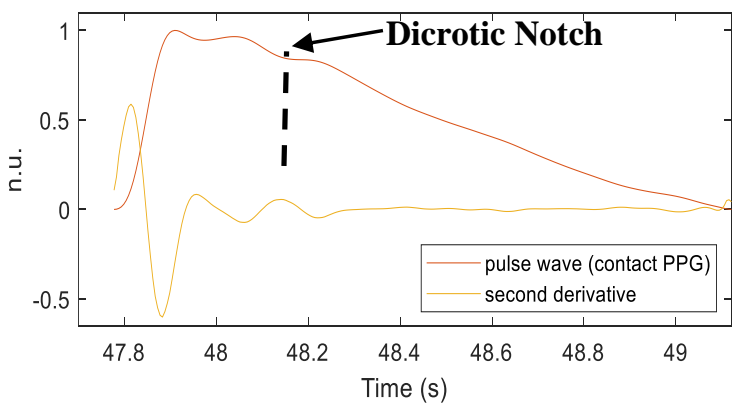

Figure 5. Contact PPG dicrotic notch detection, blue trace is the PPG waveform and the yellow trace its second derivative.

\section{Experimental setup}

The experimental setup is illustrated on Fig. 6. During the measurement, the participants were asked to sit and stay still in front of the camera at a distance of approximately $1 \mathrm{~m}$. An RGB images sequence of 60 second length was recorded. Images were saved directly as bmp format without any compression. These experiments were performed indoor and using LED light sources (Neewer NL480). For the validation of our results, an approved contact PPG measurement module was employed (see section III.A for details). PPG signal was recorded from the earlobe at a sampling rate of $256 \mathrm{~Hz}$.

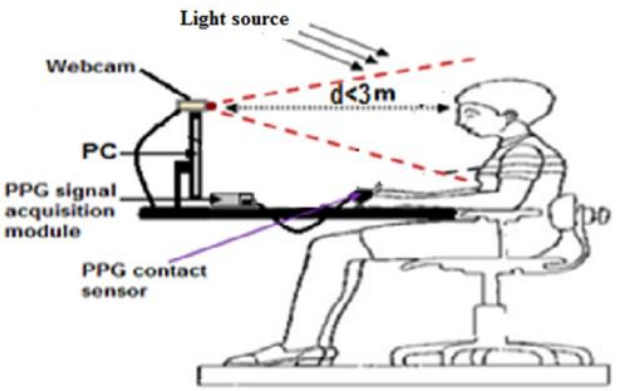

Figure 6. Experimental setup

\section{RESULTS AND DISCUSSION}

Fig. 7 shows the evolution of the extracted features (see section II) for each pulse wave. Red dashed lines correspond to the reference PPG, measured from earlobe with contact sensor, while blue solid lines to iPPG, measured on the forehead with the fast camera. The resulting traces were normalized by subtracting the mean and dividing by the standard deviation.
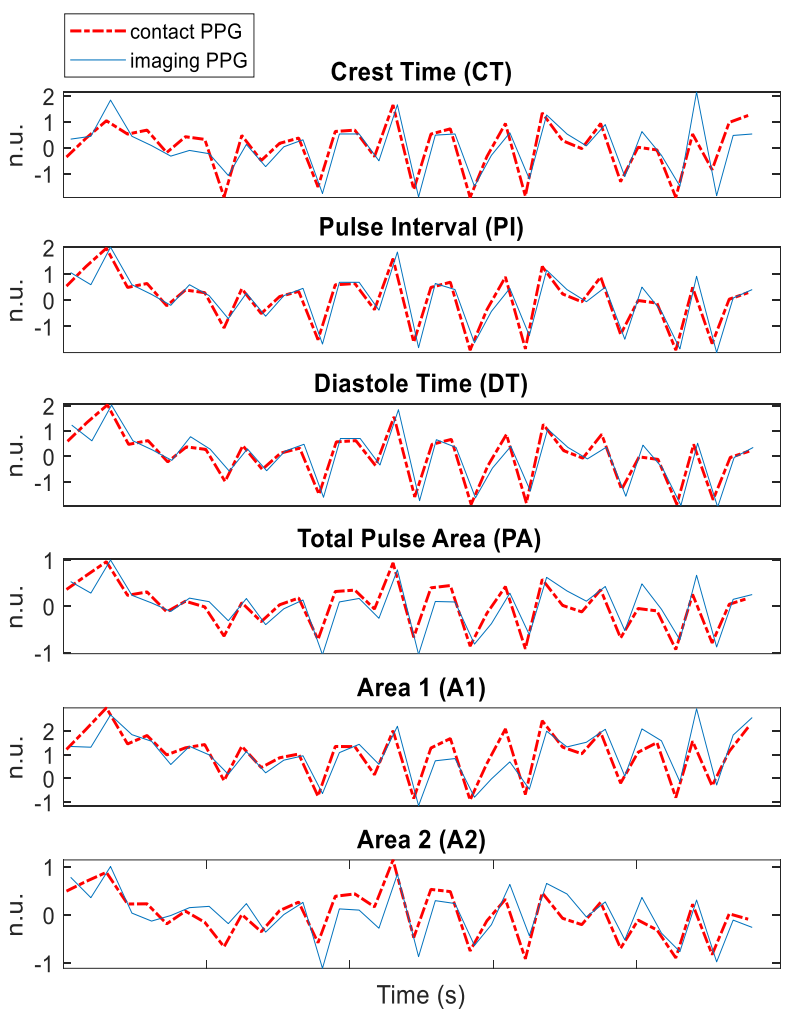

Figure 7. extracted features evolution (presented in order CT, PI, DT, TA, A1, A2), the red features traces are computed from the contact PPG and the blue traces from contactless iPPG.

iPPG waveform analysis results are shown in Fig.7 where a complete 60 seconds signal was processed without removing any distortion. The signal includes 47 successive waves that were analyzed and averaged to obtain the 37 presented values for each feature.

The obtained results exhibit high correlation with those obtained by an approved contact PPG sensor. We 
notice that time features are more correlated than areas. Pulse interval and diastolic time showed a very high correlation coefficient as given in Table I. This can allow an accurate contactless measurement of heart rate and good understanding of cardiovascular system properties as mentioned in [25]. Crest time has also shown a great stability (see Fig. 7) and high correlation coefficient. In Table I. CT values differs between both measurements. We assume that this deviation is due to the pulse wave shape and in particular to its curvature because the measurements are made on two different sites, the contact sensor being placed on earlobe while the camera measures the iPPG in the forehead area. This parameter can be used for remote evaluation and diagnosis of cardiac diseases [20].

TABLE I. STATISTICAL ANALYSIS OF THE OBTAINED FEATURES RESULTS

\begin{tabular}{|c|c|c|c|c|c|c|}
\hline \multirow{2}{*}{$\begin{array}{c}\text { FEATURE } \\
S\end{array}$} & \multicolumn{3}{|c|}{ SUBJECT 1} & \multicolumn{3}{|c|}{ SUBJECT 2} \\
\hline & $\begin{array}{l}\text { PPG } \\
\text { mean } \\
\pm \text { std } \\
\end{array}$ & $\begin{array}{c}\text { IPPG } \\
\text { mean } \\
\pm \text { std }\end{array}$ & CORR & $\begin{array}{c}\text { PPG } \\
\text { mean } \\
\pm \text { std } \\
\end{array}$ & $\begin{array}{l}\text { IPPG } \\
\text { mean } \\
\pm \text { std }\end{array}$ & CORR \\
\hline$C T(S)$ & $\begin{array}{c}\mathbf{0 . 0 9} \\
\pm 0.005\end{array}$ & $\begin{array}{c}\mathbf{0 . 3 0} \\
\pm 0.01\end{array}$ & 0.87 & $\begin{array}{c}\mathbf{0 . 1 0} \\
\pm 0.004\end{array}$ & $\begin{array}{c}\mathbf{0 . 3 1} \\
\pm 0.01\end{array}$ & 0.83 \\
\hline$P I(S)$ & $\begin{array}{c}\mathbf{1 . 3 2} \\
\pm 0.07 \\
\end{array}$ & $\begin{array}{c}\mathbf{1 . 3 2} \\
\pm 0.08 \\
\end{array}$ & 0.96 & $\begin{array}{c}\mathbf{1 . 0 7} \\
\pm 0.04 \\
\end{array}$ & $\begin{array}{c}1.07 \\
\pm 0.05\end{array}$ & 0.95 \\
\hline$D T(S)$ & $\begin{array}{c}1.22 \\
\pm 0.07\end{array}$ & $\begin{array}{c}\mathbf{1 . 0 8} \\
\pm 0.06\end{array}$ & 0.95 & $\begin{array}{c}\mathbf{0 . 9 7} \\
\pm 0.04\end{array}$ & $\begin{array}{c}\mathbf{0 . 7 5} \\
\pm 0.03\end{array}$ & 0.94 \\
\hline$P A$ & $\begin{array}{c}\mathbf{0 . 6 5} \\
\pm 0.03 \\
\end{array}$ & $\begin{array}{c}\mathbf{0 . 7 8} \\
\pm 0.04\end{array}$ & 0.89 & $\begin{array}{c}\mathbf{0 . 5 1} \\
\pm 0.02 \\
\end{array}$ & $\begin{array}{c}\mathbf{0 . 5 9} \\
\pm 0.02 \\
\end{array}$ & 0.87 \\
\hline$A 1$ & $\begin{array}{c}\mathbf{0 . 2 6} \\
\pm 0.01\end{array}$ & $\begin{array}{c}\mathbf{0 . 3 7} \\
\pm 0.25\end{array}$ & 0.86 & $\begin{array}{c}\mathbf{0 . 2 4} \\
\pm 0.01\end{array}$ & $\begin{array}{c}\mathbf{0 . 3 8} \\
\pm 0.01\end{array}$ & 0.87 \\
\hline$A 2$ & $\begin{array}{c}\mathbf{0 . 3 8} \\
\pm 0.02\end{array}$ & $\begin{array}{c}\mathbf{0 . 4 0} \\
\pm 0.02\end{array}$ & 0.84 & $\begin{array}{c}\mathbf{0 . 2 6} \\
\pm 0.01\end{array}$ & $\begin{array}{c}\mathbf{0 . 2 1} \\
\pm 0.01\end{array}$ & 0.64 \\
\hline
\end{tabular}

In addition, the presented correlation results of areas are strongly acceptable. They correspond to the most important features that can be used for several vascular diseases diagnosis, which can allow arterial stiffness and atherosclerosis assessment [29], [30] without any skin contact. Area 2 correlation equals 0.64 for subject 2 (see Table I). This drop in correlationcan be due to motion artifact.

Despite that the two waveforms are not extracted from the same ROI, they also exhibit strong correlation. These results open up prospects to use iPPG for the assessment of several cardiovascular diseases.

\section{CONCLUSION}

In this study we have developed an approach to analysis imaging photoplethysmography signal waveform. We found that the extracted waveform features such as crest time, pulse interval, diastolic time and areas are strongly correlated to those extracted from contact photoplethysmography signal. We have also quoted their potential diagnostic applications. Therefore, this made the use of iPPG more promising as contactless technonology for early screening of various atherosclerotic and cardiovascular pathologies. A non-contact diagnosis of certain vascular diseases will be the subject of our future work.

\section{REFERENCES}

[1] A. Challoner, 'Photoelectric plethysmography for estimating cutaneous blood flow', Non-Invasive Physiol. Meas., vol. 1, pp. $125-151,1979$.

[2] J. Allen, 'Photoplethysmography and its application in clinical physiological measurement', Physiol. Meas., vol. 28, no. 3, pp. R1-R39, 2007.

[3] L. A. Bortolotto, J. Blacher, T. Kondo, K. Takazawa, and M. E. Safar, 'Assessment of vascular aging and atherosclerosis in hypertensive subjects: second derivative of photoplethysmogram versus pulse wave velocity', Am. J. Hypertens., vol. 13, no. 2, pp. 165-171, Feb. 2000.

[4] N. Miyai, K. Miyashita, M. Arita, I. Morioka, K. Kamiya, and S. Takeda, 'Noninvasive assessment of arterial distensibility in adolescents using the second derivative of photoplethysmogram waveform', Eur. J. Appl. Physiol., vol. 86, no. 2, pp. 119-124, Dec. 2001.

[5] F. Bousefsaf, C. Maaoui, and A. Pruski, 'Peripheral vasomotor activity assessment using a continuous wavelet analysis on webcam photoplethysmographic signals', Biomed. Mater. Eng., vol. 27, no. 5, pp. 527-538, Nov. 2016.

[6] M.-Z. Poh, D. J. McDuff, and R. W. Picard, 'Non-contact, automated cardiac pulse measurements using video imaging and blind source separation.', Opt. Express, vol. 18, no. 10, pp. 10762-10774, 2010.

[7] F. Bousefsaf, C. Maaoui, and A. Pruski, 'Continuous wavelet filtering on webcam photoplethysmographic signals to remotely assess the instantaneous heart rate', Biomed. Signal Process. Control, vol. 8, no. 6, pp. 568-574, 2013.

[8] M.-Z. Poh, D. J. McDuff, and R. W. Picard, 'Advancements in Noncontact, Multiparameter Physiological Measurements Using a Webcam', IEEE Trans. Biomed. Eng., vol. 58, no. 1, pp. 7-11, Jan. 2011.

[9] F. Bousefsaf, C. Maaoui, and A. Pruski, 'Remote detection of mental workload changes using cardiac parameters assessed with a low-cost webcam', Comput. Biol. Med., vol. 53, pp. 154 163, Oct. 2014.

[10] D. Shao et al., 'Noncontact monitoring of blood oxygen saturation using camera and dual-wavelength imaging system', IEEE Trans. Biomed. Eng., vol. 63, no. 6, pp. 1091-1098, 2016.

[11] A. R. Guazzi et al., 'Non-contact measurement of oxygen saturation with an RGB camera', Biomed. Opt. Express, vol. 6, no. 9 , p. 3320 , Sep. 2015.

[12] U. Bal, 'Non-contact estimation of heart rate and oxygen saturation using ambient light', Biomed. Opt. Express, vol. 6, no. 1, p. 86, Jan. 2015.

[13] I. C. Jeong and J. Finkelstein, 'Introducing contactless blood pressure assessment using a high speed video camera', J. Med. Syst., vol. 40, no. 4, p. 77, 2016.

[14] N. Sugita, M. Yoshizawa, M. Abe, A. Tanaka, N. Homma, and T. Yambe, 'Contactless Technique for Measuring BloodPressure Variability from One Region in Video Plethysmography', J. Med. Biol. Eng., pp. 1-10, 2018.

[15] X. Fan, Q. Ye, X. Yang, and S. D. Choudhury, 'Robust blood pressure estimation using an RGB camera', J. Ambient Intell. Humaniz. Comput., Sep. 2018.

[16] M. Elgendi, 'On the analysis of fingertip photoplethysmogram signals', Curr. Cardiol. Rev., vol. 8, no. 1, pp. 14-25, 2012.

[17] A. Reisner, P. A. Shaltis, D. McCombie, and H. H. Asada, 'Utility of the photoplethysmogram in circulatory monitoring', Anesthesiol. J. Am. Soc. Anesthesiol., vol. 108, no. 5, pp. 950958, 2008.

[18] L. Nilsson, A. Johansson, and S. Kalman, 'Respiration can be monitored by photoplethysmography with high sensitivity and specificity regardless of anaesthesia and ventilatory mode', Acta Anaesthesiol. Scand., vol. 49, no. 8, pp. 1157-1162, 2005. 
[19] K. Takazawa et al., 'Assessment of vasoactive agents and vascular aging by the second derivative of photoplethysmogram waveform', Hypertension, vol. 32, no. 2, pp. 365-370, 1998.

[20] S. R. Alty, N. Angarita-Jaimes, S. C. Millasseau, and P. J. Chowienczyk, 'Predicting arterial stiffness from the digital volume pulse waveform', IEEE Trans. Biomed. Eng., vol. 54, no. 12, pp. 2268-2275, 2007.

[21] A. Wang et al., 'Quantification of radial arterial pulse characteristics change during exercise and recovery', J. Physiol. Sci., vol. 68, no. 2, pp. 113-120, 2018.

[22] H.-T. Wu et al., 'Novel application of parameters in waveform contour analysis for assessing arterial stiffness in aged and atherosclerotic subjects', Atherosclerosis, vol. 213, no. 1, pp. 173-177, Nov. 2010.

[23] A. Wang et al., 'Athletic differences in the characteristics of the photoplethysmographic pulse shape: effect of maximal oxygen uptake and maximal muscular voluntary contraction', BioMed Res. Int., vol. 2015, 2015.

[24] M. S. Tanveer and M. K. Hasan, 'Cuffless blood pressure estimation from electrocardiogram and photoplethysmogram using waveform based ANN-LSTM network', Biomed. Signal Process. Control, vol. 51, pp. 382-392, 2019.

[25] C. Poon, X. Teng, Y. Wong, C. Zhang, and Y. Zhang, 'Changes in the photoplethysmogram waveform after exercise', in 2004 2nd IEEE/EMBS International Summer School on Medical Devices and Biosensors, 2004, pp. 115-118.

[26] S. Lu et al., 'Can Photoplethysmography Variability Serve as an Alternative Approach to Obtain Heart Rate Variability Information?', J. Clin. Monit. Comput., vol. 22, no. 1, pp. 2329, Jan. 2008
[27] K. Zhang, Y. Wang, S. Zhang, Y. Yang, Z. Luo, and Y. Zeng, 'Dynamic analysis for blood flow parameters of cardiovascular system on work load', in 2005 IEEE Engineering in Medicine and Biology 27th Annual Conference, 2006, pp. 5551-5554.

[28] E. Seitsonen et al., 'EEG spectral entropy, heart rate, photoplethysmography and motor responses to skin incision during sevoflurane anaesthesia', Acta Anaesthesiol. Scand., vol. 49, no. 3, pp. 284-292, 2005.

[29] P. Salvi, C. Palombo, G. M. Salvi, C. Labat, G. Parati, and A. Benetos, 'Left ventricular ejection time, not heart rate, is an independent correlate of aortic pulse wave velocity', J. Appl. Physiol., vol. 115, no. 11, pp. 1610-1617, Dec. 2013.

[30] C.-M. Huang et al., 'Radial Pressure Pulse and Heart Rate Variability in Heat- and Cold-Stressed Humans', Evid. Based Complement. Alternat. Med., vol. 2011, pp. 1-9, 2011.

[31] F. Bousefsaf, C. Maaoui, and A. Pruski, 'Automatic Selection of Webcam Photoplethysmographic Pixels Based on Lightness Criteria', J. Med. Biol. Eng., vol. 37, no. 3, pp. 374-385, Jun. 2017.

[32] G. Lempe, S. Zaunseder, T. Wirthgen, S. Zipser, and H. Malberg, 'ROI Selection for Remote Photoplethysmography', in Bildverarbeitung für die Medizin 2013, H.-P. Meinzer, T. M Deserno, H. Handels, and T. Tolxdorff, Eds. Berlin, Heidelberg: Springer Berlin Heidelberg, 2013, pp. 99-103.

[33] B. L. Horecker, 'The absorption spectra of hemoglobin and its derivatives in the visible and near infra-red regions', $J$ Biol Chem, vol. 148, no. 1, pp. 173-183, 1943.

[34] M. P. Tarvainen, P. O. Ranta-aho, and P. A. Karjalainen, 'An advanced detrending method with application to HRV analysis', IEEE Trans. Biomed. Eng., vol. 49, no. 2, pp. 172-175, Feb. 2002 